\section{Fibromyalgie: TENS lindert bewegungsinduzierte Schmerzen und Fatigue}

Dailey DL et al. Transcutaneous Electrical Nerve Stimulation Reduces Movement-Evoked Pain and Fatigue: A Randomized, Controlled Trial. Arthritis Rheumatol 2020; 72: 824-836. doi: 10.1002/ art.41170

Patienten mit einer Fibromyalgie klagen über generalisierte Schmerzen, insbesondere während körperlicher Aktivitäten. Da die transkutane elektrische Nervenstimulation (TENS) endogene Analgesiemechanismen aktiviert, untersuchten US-Forscher, wie gut dieses Therapieverfahren bewegungsinduzierte Schmerzen lindert und sich günstig auf weitere Fibromyalgie-Beschwerden wie Erschöpfungszustände auswirkt.

An der randomisierten Phase II-Studie nahmen 301 Frauen mit einer Fibromyalgie teil, welche seit mindestens 4 Wochen unter einer stabilen Medikation standen. $103 \mathrm{~Pa}$ tientinnen wendeten über 4 Wochen mindestens 2 Stunden pro Tag während körperlicher Aktivitäten TENS in der maximal tolerierbaren Intensität an. Die Elektroden platzierten die Frauen dabei am zervikothorakalen Übergang sowie am unteren Rücken. Weitere 99 Patientinnen erhielten eine TENS-Scheinintervention und 99 Frauen erhielten keine TENS. Vor und nach der TENS-Anwendung quantifizierten die Studienteilnehmerinnen die Schmerzintensität in Ruhe bzw. während der Bewegung (primärer Studienendpunkt) sowie die Fatigue mithilfe einer 11 Punkte umfassenden numerischen Bewertungsskala. Weitere sekundäre Outcome-Parameter umfassten unter anderem die körperliche Funktion, die Angst vor Bewegungen, die Auswirkungen der Erkrankung, die Lebensqualität sowie weitere psychische Parameter.

\section{Ergebnisse}

Die Studienteilnehmerinnen wendeten die aktive bzw. die Placebo-TENS im Median über 77 bzw. 72 Min. pro Tag an. Nach der 4-wöchigen Interventionsphase berichteten die mit TENS behandelten Frauen sowohl im Vergleich zur Placebo- als auch zur Kontrollgruppe über eine signifikante Abnahme bewegungsinduzierter Schmerzen. Auch im Hinblick auf die Schmerzen in Ruhe sowie die bewegungsinduzierte Fatigue erwies sich die aktive TENS der Scheinintervention sowie der Nicht-Intervention als signifikant überlegen. Ferner berichteten $70 \%$ der mit TENS aber nur 31\% der mit Placebo und $9 \%$ der Kontrollen (jeweils $p<0,0001$ ) über eine allgemeine Zustandsverbesserung (Global Impression of Change). Die aktive TENS-Behandlung ging zudem im Vergleich zur Nicht-Behandlung, nicht jedoch im Vergleich zu Placebo mit einer signifikanten Verbesserung der Krankheitsauswirkungen sowie der subjektiven Funktion einher. Schwere Nebenwirkungen traten unter den TENS-Interventionen nicht auf. Weniger als $5 \%$ der Studienteilnehmerinnen berichteten über leichte Nebenwirkungen wie Schmerzen oder Hautirritationen.
FAZIT

Stabil auf eine Medikation eingestellte Fibromyalgiepatientinnen profitieren hinsichtlich bewegungsinduzierter Schmerzen, Erschöpfungszustände sowie weiterer klinischer Parameter von einer TENS-Behandlung, schlussfolgern die Autoren. Weitere Studien müssen diese Ergebnisse nun bestätigen, ihre klinische Relevanz beleuchten und klären, wie lange der schmerzlindernde Effekt der Therapie anhält.

Dr. med. Judith Lorenz, Künzell 